\title{
Diagnostic value of the ultrasonographic description of a splenic mass or nodule as cavitated in 106 dogs with nontraumatic hemoabdomen
}

\author{
Stephen L. Millar DVM \\ Kristin M. Zersen DVM
}

From the Department of Clinical Sciences, College of Veterinary Medicine and Biomedical Sciences, Colorado State University, Fort Collins, CO 80523.

Address correspondence to Dr. Zersen (kristin.zersen@colostate.edu)

\author{
OBJECTIVE \\ To assess the diagnostic value of the ultrasonographic description of a splenic \\ mass or nodule as cavitated in dogs with nontraumatic hemoabdomen.
}

\section{ANIMALS}

106 dogs with a nontraumatic hemoabdomen that underwent abdominal ultrasonography and splenectomy with histologic examination of splenic lesions between 2005 and 2018 .

\section{PROCEDURES}

Medical records were reviewed for abdominal ultrasonographic and histologic findings. Diagnostic performance of ultrasonographic description of a splenic mass or nodule as cavitated as evidence of hemangiosarcoma or any malignancy was evaluated.

\begin{abstract}
RESULTS
Ultrasonographic description of splenic lesions as cavitated had poor diagnostic utility in predicting presence of hemangiosarcoma or malignancy. Sensitivity and specificity of this test were $41.9 \%$ (95\% Cl, $30.5 \%$ to $54.3 \%$ ) and $51.2 \%(95 \% \mathrm{Cl}, 36.8 \%$ to $65.4 \%)$, respectively, for detecting hemangiosarcoma, with positive and negative predictive values of $55.3 \%(95 \% \mathrm{Cl}$, $41.2 \%$ to $68.6 \%)$ and $37.9 \%(95 \% \mathrm{Cl}, 26.6 \%$ to $50.8 \%)$, respectively. Results were similar for detecting malignancy. Cavitated lesions outside of the spleen were too rare for statistical analysis to be of value.
\end{abstract}

\section{CONCLUSIONS AND CLINICAL RELEVANCE}

Results suggested that relying on ultrasonographic description of cavitation to diagnose splenic lesions as malignant in dogs with nontraumatic hemoabdomen is unfounded. Other preoperative diagnostic tests may be more valuable in determining short- and long-term prognoses.
$\mathrm{T}$ There is a stark difference in the prognosis for dogs with malignant versus nonmalignant causes of spontaneous nontraumatic hemoabdomen, also called hemoperitoneum. ${ }^{1-9}$ The most common cause of nontraumatic hemoabdomen in dogs is neoplasia, which can be malignant or benign. ${ }^{10}$ However, most neoplastic splenic lesions in dogs are malignant and the vast majority of those lesions are hemangiosarcoma. ${ }^{10}$

Survival time for dogs with splenic hemangiosarcoma that undergo surgery alone is typically 1 to 3 months. ${ }^{2,4}$ In contrast, dogs with benign splenic lesions have a median survival time of 21 to 22 months. ${ }^{8,9}$ Typically, in dogs, nontraumatic hemoabdomen is a result of rupture of a splenic mass or nodule, ${ }^{1,10}$ but other causes of nontraumatic hemoabdomen that have been reported include coagulopathy, gastric dilatation-volvulus, splenic torsion, liver lobe torsion, and anaphylaxis. ${ }^{1,11,12}$ After a diagnosis of nontraumatic hemoabdomen is made, clinicians and owners are faced with making decisions regarding treatment in the face of substantial uncertainty regarding the eventual prognosis. ${ }^{11}$

Most causes of hemoabdomen unrelated to an abdominal mass or nodule can be ruled out prior to surgery with appropriate diagnostic imaging, biochemical testing, and coagulation testing. ${ }^{1,10,11}$ Efforts have been made to help distinguish malignant from nonmalignant masses and nodules prior to surgery. Recently, the hemangiosarcoma likelihood predictor score has been described as a means to predict the likelihood of hemangiosarcoma versus hematoma..$^{13}$ This score is calculated on the basis of body weight, total solids concentration, platelet count, and radiographic lung pattern to assign a low, medium, or high risk of hemangiosarcoma..$^{13} \mathrm{~A}$ higher mass-to-splenic volume ratio and higher splenic weight as a percentage of body weight are also suggestive of benign splenic disease. ${ }^{14}$ Efforts have also been made to distinguish malignant from nonmalignant lesions through the use of contrast-enhanced ultrasonography, CT, and MRI, but these methods are insufficiently accurate, too expensive, or too time-consuming to be used in an emergency situation for dogs with hemoabdomen. ${ }^{10,11,15-17}$ The gold standard test is histologic examination of a tissue sample, which can only be obtained surgically in dogs with emergent hemoabdomen.,10,14,16-19 Diagnostic abdominal ultrasonography, which can help with surgical planning by providing confirmation of a mass or nodule, is often the most advanced diagnostic imaging pursued prior to the decision of whether to perform surgery is made. 
Ultrasonographic identification of a cavitated splenic mass or nodule has anecdotally been associated with malignancy in dogs with nontraumatic hemoabdomen; however, rigorous evidence-based findings to support this association have not been published. Ultrasonography of the spleen has been recommended for disease localization, to differentiate cavitary from solid lesions, and to assist in aspiration of lesions, ${ }^{20}$ and even point-of-care ultrasonography guidelines suggest looking for cavitations. ${ }^{21,22}$ Splenic hemangiosarcoma has been described to generally appear as a complex mass lesion with mixed echogenicity and cavitations, ${ }^{19}$ but a definitive diagnosis can only be made through histologic examination. ${ }^{19}$ Terms such as cavitary, cavitated, and the like have commonly been used to describe the ultrasonographic appearance of abdominal lesions. ${ }^{3,19-28}$ Other ultrasonographic patterns such as honeycomb patterns and target lesions have been assessed for their association with malignant hepatic and splenic lesions in dogs and cats and have been shown to have some degree of diagnostic utility. ${ }^{29-32}$

Although terms such as cavitated are commonly used to describe the ultrasonographic appearance of splenic lesions, the diagnostic performance of this description in the identification of hemangiosarcoma or other malignancies in dogs with nontraumatic hemoabdomen has not been evaluated. The percentage of dogs with ultrasonographic evidence of rupture of a splenic mass that are euthanized without further treatment has not been established but is likely high because of the potential that these masses are malignant. In 1 study, ${ }^{33}$ about $80 \%$ of dogs with hemoabdomen of all causes died, were euthanized, or did not require surgery. Evaluating the relationship between ultrasonographic identification of a cavitated splenic mass and malignancy would help clinicians and owners make better-informed decisions or could help reduce inaccurate clinical prognoses. The objective of the study reported here was to assess the diagnostic value of the ultrasonographic description of a splenic mass or nodule as cavitated in dogs with nontraumatic hemoabdomen. On the basis of our clinical experience, we hypothesized that ultrasonographic identification of a cavitated splenic mass or nodule in dogs with nontraumatic hemoabdomen would not accurately predict a diagnosis of hemangiosarcoma or other malignancy.

\section{Materials and Methods}

\section{Animals}

The electronic medical record system of the Colorado State University Veterinary Teaching Hospital was searched to identify all dogs charged for a splenectomy performed between January 2005 and December 2018. Dogs were eligible for inclusion in the study if they had undergone abdominal ultrasonography performed by a diplomate of the American College of Veterinary Radiology, had a splenic mass or nodule, and had evidence of blood in the peritoneal cavity $<2$ weeks prior to surgery. Dogs were excluded if a splenic mass or nodule and hemoabdomen were not documented; if concurrent gastric-dilatation volvulus, septic or bile peritonitis, splenic torsion or abscess, or trauma was documented; the medical record was incomplete; or results of histologic examination of splenic tissue were not available.

\section{Data collection}

For dogs included in the study, information obtained from the electronic medical record consisted of signalment (ie, sex, neuter status, and age) and results of abdominal ultrasonography, including whether the splenic mass or nodule was described as cavitated and whether lesions were identified in the liver, mesentery, peritoneal space, or elsewhere in the abdomen. In instances when ultrasonographic images were available for review, a diplomate of the American College of Veterinary Emergency and Critical Care reviewed all available images for any clear discrepancies in mass description. At the time of the study, the routine procedure for dogs undergoing splenectomy at our institution was to submit the entire spleen for histologic examination, which was performed by diplomates of the American College of Veterinary Pathology. For the present study, results of histologic examination of splenic tissue were classified as hemangiosarcoma, nonhemangiosarcoma malignant, nonmalignant, or normal.

\section{Statistical analysis}

Distributions of age and weight were tested for normalcy with the Anderson-Darling test. Continuous data that were normally distributed were summarized as mean $\pm \mathrm{SD}$; continuous data that were not normally distributed were summarized as median and range. Results of histologic examination of splenic tissue were considered the gold standard for diagnostic status. Lesions identified by means of abdominal ultrasonography and for which results of histologic examination were available were classified as splenic, hepatic, or mesenteric and other. The diagnostic test evaluated in the present study was whether a mass or nodule detected during abdominal ultrasonography was described as cavitated or not. The test result was considered positive if the mass or nodule was described as cavitated and negative if the mass or nodule was not described as cavitated. Sensitivity, specificity, positive predictive value (PPV), and negative predictive value (NPV), along with their 95\% CIs, were then calculated both for detecting hemangiosarcoma and for detecting any malignancy, including hemangiosarcoma. Sensitivity was calculated as the percentage of dogs that had a positive test result among dogs with hemangiosarcoma or among dogs with a malignant mass or nodule detected by abdominal ultrasonography. Specificity was calculated as the percentage of dogs that had a negative test result among dogs that did not have hemangiosarcoma or 
among dogs that had a nonmalignant mass or nodule detected by abdominal ultrasonography. The PPV was calculated as the percentage of dogs with hemangiosarcoma or any malignant mass or nodule among all dogs with positive test results. The NPV was calculated as the percentage of dogs that did not have hemangiosarcoma or any malignancy among all dogs with negative test results. Sensitivity, specificity, PPV, and NPV were calculated separately for the splenic, hepatic, and mesenteric or other lesions when sufficient numbers of cases were present. Notably, not every lesion detected by means of abdominal ultrasonography was sampled, and some lesions were identified during surgery without first being identified by means of preoperative abdominal ultrasonography. All statistical analyses were performed with standard software (JMP version 15.0.0; SAS Institute Inc) with the performance measures add-in.

\section{Results}

A total of 701 dogs underwent splenectomy during the study period; however, 595 dogs were excluded because of coexisting medical conditions, a lack of histologic examination of splenic tissues, a lack of abdominal ultrasonography, or incomplete medical records. The remaining 106 dogs were included in the study.

Of the 106 dogs included in the study, 54 (50.9\%) were male and 52 (49.1\%) were female. Only 8 (7.5\%) of the 106 dogs were sexually intact. Median age at the time of splenectomy was 11.1 years (range, 2.7 to 15.4 years), and mean \pm SD weight was $28.5 \pm 11.4$ $\mathrm{kg}$. Sixty-two (58.5\%) of the 106 dogs had hemangiosarcoma, and 74 (69.8\%) had a malignancy (including hemangiosarcoma).

A total of 147 masses or nodules (105 splenic, 27 hepatic, and 15 mesenteric or other) were detected by abdominal ultrasonography, of which 51 (34.7\%) were described as cavitated (Figures I-3); 207 masses or nodules were identified during surgery (106 splenic, 58 hepatic, and 43 mesenteric or other). Two hundred thirty samples were submitted for histologic examination. Sixty-two (58.5\%) of the 106 splenic tissue

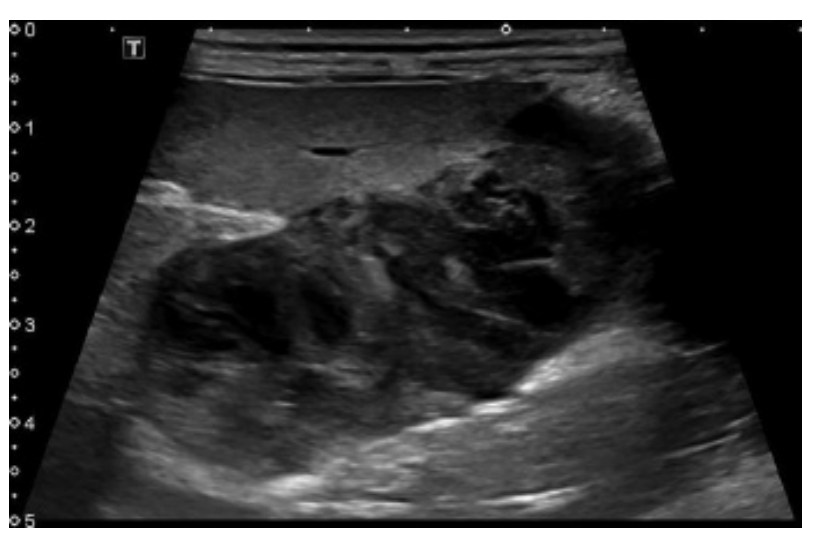

Figure I-Ultrasonographic image of a cavitated splenic lesion in a 12-year-old terrier; the histologic diagnosis was hemangiosarcoma. samples were hemangiosarcoma, and 73 (68.9\%) were classified as a malignancy (including hemangiosarcoma). Twenty-nine (23.4\%) of the 124 nonsplenic tissue samples were identified histologically as hemangiosarcoma, and 39 (31.5\%) were identified as a malignancy.

A total of 105 dogs were found to have a splenic mass or nodule on abdominal ultrasonography, of which 47 (44.8\%) had cavitated lesions, and 62 (58.5\%) of the 105 dogs were determined to have hemangiosarcoma on the basis of histologic examination of splenic tissues. Sensitivity, specificity, PPV, and NPV of ultrasonographic detection of a cavitated splenic mass or nodule as a test for hemangiosarcoma or as a test for any malignancy were generally low. As a test for hemangiosarcoma, sensitivity of ultrasonographic detection of a cavitated splenic mass or nodule was $41.9 \%$ (26/62; 95\% CI, 30.5\% to $54.3 \%)$ and specificity was $51.2 \%(22 / 43 ; 95 \% \mathrm{CI}, 36.8 \%$ to $65.4 \%)$, with a PPV of $55.3 \%(26 / 47 ; 95 \% \mathrm{CI}, 41.2 \%$ to $68.6 \%)$ and NPV of $37.9 \%$ (22/58; $95 \%$ CI, $26.6 \%$ to $50.8 \%)$.

Similarly, 105 dogs were found to have a splenic mass or nodule on abdominal ultrasonography, including 47 (44.8\%) with cavitated lesions, and 73 (68.9\%) were determined to have a malignancy, including hemangiosarcoma, on the basis of histologic examination of splenic tissues. As a test for malignancy, sensitivity of ultrasonographic detection of a cavitated splenic mass or nodule was 39.7\% (29/73; 95\% CI, $29.3 \%$ to $51.2 \%)$, specificity was $43.8 \%(14 / 32$; $95 \%$ CI, $28.2 \%$ to $60.7 \%)$, PPV was $61.7 \%$ (29/47; 95\% CI, $47.4 \%$ to $74.2 \%)$, and NPV was $24.1 \%(14 / 58 ; 95 \%$ CI, $15.0 \%$ to $36.5 \%)$.

Twenty-seven of the 106 (25.5\%) dogs were found to have a hepatic mass or nodule on abdominal ultrasonography, and 2 (7.4\%) of the 27 lesions were cavitated. Of the 79 dogs found to have a hepatic mass or nodule by abdominal ultrasonography or surgery (or both), 16 (20.3\%) had hemangiosarcoma and 21 (26.6\%) had a malignancy, including hemangiosarcoma.

Finally, 15 (14.2\%) of the 106 dogs were found to have a mass or nodule involving the mesentery or other abdominal structure on abdominal ultrasonography,

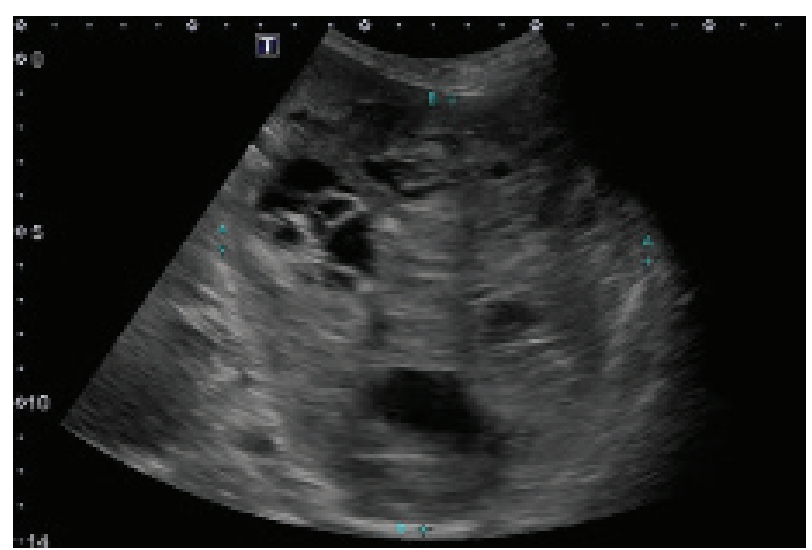

Figure 2-Ultrasonographic image of a cavitated splenic lesion in a 15-year-old Pointer; the histologic diagnosis was hematoma. 


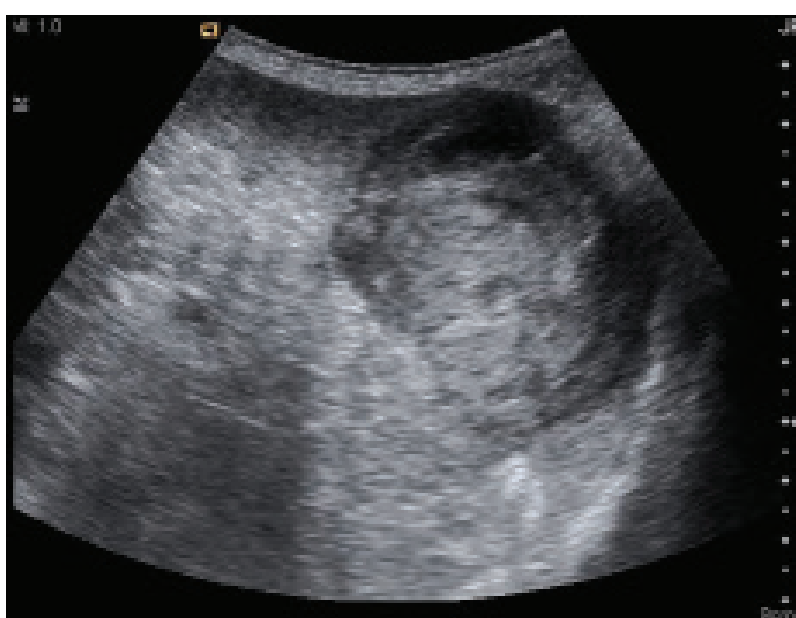

Figure 3-Ultrasonographic image of a noncavitated splenic lesion in a 9-year-old Labrador; the histologic diagnosis was hemangiosarcoma.

and $2(13.3 \%)$ of the 15 lesions were cavitated. Of the 45 dogs found to have a mesenteric or other mass or nodule by abdominal ultrasonography or surgery (or both), $13(28.9 \% \%)$ had hemangiosarcoma and 18 (40.0\%) had a malignancy, including hemangiosarcoma.

There were too few dogs with hepatic masses or nodules or mesenteric or other masses or nodules to reliably calculate sensitivity, specificity, PPV, or NPV. Histologic diagnoses for the 18 dogs with splenic masses or nodules that were described as cavitated on abdominal ultrasonography but were not malignant on histologic examination were hematoma $(n=10)$, hematoma and lymphoid hyperplasia (2), myelolipoma with hemorrhage or rupture (2), hemangioma and hematoma (2), hematoma and extramedullary hematopoiesis (1), and lymphoid hyperplasia with hemorrhage (1).

\section{Discussion}

Results of the present study, as suspected, did not provide any evidence to support the use of detection of a cavitated lesion during abdominal ultrasonography as an indicator of hemangiosarcoma or other malignancy in dogs. For splenic lesions in particular, the sensitivity, specificity, PPV, and NPV were all low, making the test unreliable. There were not enough cases to properly evaluate diagnostic utility of detecting cavitated nonsplenic masses or nodules, but only 4 (3.8\%) of 106 dogs in our study had a cavitated nonsplenic mass or nodule. Further multicenter studies could likely provide additional information for this population. However, our findings suggest that abdominal ultrasonographic examination for the purpose of distinguishing malignant from nonmalignant splenic or nonsplenic lesions in dogs with nontraumatic hemoabdomen will likely not be helpful. On the other hand, abdominal ultrasonography in dogs with nontraumatic hemoabdomen may be helpful in surgical planning and confirming the presence of a bleeding mass or nodule. This could be especially important for clinicians who have the equipment and experience necessary to perform a splenec- tomy but not a liver lobectomy. And, should the source of bleeding be elsewhere, such as the adrenal glands, kidneys, or gastrointestinal system, preoperative abdominal ultrasonography could be quite valuable in planning and prognostication. To our knowledge, it is not yet known how results of abdominal ultrasonography specifically influence a clinician's decision on how or whether to perform surgery. In a previous study, ${ }^{34}$ $>50 \%$ of dogs with nontraumatic hemoabdomen that underwent preoperative abdominal ultrasonography had a grossly detectable lesion at surgery that was not found with ultrasonography. And, in a study ${ }^{35}$ in which isolated hepatic masses were detected ultrasonographically, approximately half were not correctly matched to their true lobe or division. In any case, inappropriate attribution of malignancy on the basis of results of preoperative abdominal ultrasonography could lead to unjustified euthanasia of dogs with benign disease and a good prognosis.

The present study had several limitations affecting the impact of our findings. This was a single-center retrospective study, and criteria for identifying which lesions were cavitated were not standardized among radiologists. When only still images are available, they may not fully represent the lesion, and about a third (33/106) of dogs did not have imaging data to review. Individual radiologists may use the term cavitated at different rates or may have differing personal criteria for what constitutes cavitated. Nevertheless, in the present study, 57 (53.8\%) of the 106 dogs had at least 1 lesion described as cavitated, illustrating the pervasive use of this term in radiologists' reports. Despite this, to our knowledge, there is not a defined set of criteria for this pattern description. A prospective multicenter study incorporating defined criteria for cavitation or testing radiologists' gestalt assessments of malignancy may help extend the evaluation of this topic. Another limitation of the present study was that, although histologic examination is the gold standard, it can be difficult to thoroughly examine every aspect of the spleen.,10,14,16-19 The standard at our institution is to submit the spleen in its entirety for histologic evaluation by a pathologist. Finally, the present study's population was relatively small and only included dogs with ruptured lesions (ie, nontraumatic hemoabdomen). This population was chosen because dogs with nontraumatic hemoabdomen are often not stable enough to perform extensive diagnostic testing prior to surgery, requiring that a decision to proceed with treatment or euthanasia be made with limited information. In these time-sensitive situations, overreliance on the minimal amount of data available could lead to inaccurate decisions. Importantly, whether the present study's findings are similar to those for dogs with abdominal lesions but without hemoabdomen is not known.

In the present study, we only included dogs that underwent surgery, and therefore, our population may not represent the entire group of dogs with nontraumatic hemoabdomen. Many dogs with nontraumatic hemoabdomen are euthanized without undergoing surgery and 
do not have a necropsy performed. Therefore, the true percentage of dogs with nontraumatic hemoabdomen that have benign disease is unknown. Regardless, for dogs in the present study, there was no evidence to support a diagnosis of malignancy on the basis of ultrasonographic detection of a cavitated lesion, and such a finding should not influence any recommendation or decision regarding euthanasia. Other preoperative tests, such as thoracic radiography, hemangiosarcoma likelihood predictor scoring, and contrast-enhanced imaging, may be of higher clinical value in predicting the presence of malignant lesions in this population of dogs.

\section{Acknowledgments}

No third-party funding or support was received in connection with this study or the writing or publication of the manuscript. The authors declare that there were no conflicts of interest.

\section{References}

1. Pesillo-Crosby SA. Hemoperitoneum. In: Drobatz KJ, Hopper K, Rozanski EA, Silverstein DC, eds. Textbook of Small Animal Emergency Medicine. Wiley-Blackwell; 2019:528-534.

2. Wendelburg KM, Price LL, Burgess KE, Lyons JA, Lew FH, Berg J. Survival time of dogs with splenic hemangiosarcoma treated by splenectomy with or without adjuvant chemotherapy: 208 cases (2001-2012). J Am Vet Med Assoc. 2015;247(4):393-403.

3. Mullin C, Clifford CA. Miscellaneous tumors. In: Vail DM Thamm DH, Liptak JM, eds. Withrow and MacEwen's Small Animal Clinical Oncology. 6th ed. Elsevier; 2020:773-810.

4. Wood CA, Moore AS, Gliatto JM, Ablin LA, Berg RJ, Rand WM. Prognosis for dogs with stage I or II splenic hemangiosarcoma treated by splenectomy alone: 32 cases (1991-1993). J Am Anim Hosp Assoc. 1998;34(5):417-421.

5. Spangler WL, Kass PH. Pathologic factors affecting postsplenectomy survival in dogs. J Vet Intern Med. 1997;11(3):166-171.

6. Weinstein MJ, Carpenter JL, Schunk CJ. Nonangiogenic and nonlymphomatous sarcomas of the canine spleen: 57 cases (1975-1987). J Am Vet Med Assoc. 1989;195(6):784-788.

7. Spangler WL, Culbertson MR. Prevalence, type, and importance of splenic diseases in dogs: 1,480 cases (1985-1989). $J$ Am Vet Med Assoc. 1992;200(6):829-834.

8. Prymak C, McKee L, Goldschmidt M, Glickman LT. Epidemiologic, clinical, pathologic, and prognostic characteristics of splenic hemangiosarcoma and splenic hematoma in dogs: 217 cases (1985). J Am Vet Med Assoc. 1988;193(6):706-712.

9. Patten SG, Boston SE, Monteith GJ. Outcome and prognostic factors for dogs with a histological diagnosis of splenic hematoma following splenectomy: 35 cases (2001-2013). Can Vet J. 2016;57(8):842-846.

10. Pintar J, Breitschwerdt EB, Hardie EM, Spaulding KA. Acute nontraumatic hemoabdomen in the dog: a retrospective analysis of 39 cases (1987-2001). J Am Anim Hosp Assoc. 2003;39(6):518-522.

11. Brockman DJ, Mongil CM, Aronson LR, Brown DC. A practical approach to hemoperitoneum in the dog and cat. Vet Clin North Am Small Anim Pract. 2000;30(3):657-668.

12. Caldwell DJ, Petras KE, Mattison BL, Wells RJ, Heffelman VL. Spontaneous hemoperitoneum and anaphylactic shock associated with Hymenoptera envenomation in a dog. J Vet Emerg Crit Care (San Antonio). 2018;28(5):476-482.

13. Schick AR, Hayes GM, Singh A, Mathews KG, Higginbotham ML, Sherwood JM. Development and validation of a hemangiosarcoma likelihood prediction model in dogs presenting with spontaneous hemoabdomen: the HeLP score. $J$ Vet Emerg Crit Care (San Antonio). 2019;29(3):239-245.

14. Mallinckrodt MJ, Gottfried SD. Mass-to-splenic volume ratio and splenic weight as a percentage of body weight in dogs with malignant and benign splenic masses: 65 cases (20072008). J Am Vet Med Assoc. 2011;239(10):1325-1327.
15. Hammond TN, Pesillo-Crosby SA. Prevalence of hemangiosarcoma in anemic dogs with a splenic mass and hemoperitoneum requiring a transfusion: 71 cases (2003-2005). J Am Vet Med Assoc. 2008;232(4):553-558.

16. Ivan i M, Long F, Seiler GS. Contrast harmonic ultrasonography of splenic masses and associated liver nodules in dogs. $J$ Am Vet Med Assoc. 2009;234(1):88-94.

17. Shanaman MM, Schwarz T, Gal A, O'Brien RT. Comparison between survey radiography, B-mode ultrasonography, contrastenhanced ultrasonography and contrast-enhanced multi-detector computed tomography findings in dogs with acute abdominal signs. Vet Radiol Ultrasound. 2013;54(6):591-604.

18. Grimes JA, Prasad N, Levy S, et al. A comparison of microRNA expression profiles from splenic hemangiosarcoma, splenic nodular hyperplasia, and normal spleens of dogs. BMC Vet Res. 2016;12(1):272. doi:10.1186/s12917-016-0903-5

19. Lisciandro S. Focused or COAST ${ }^{3}-$ spleen. In: Lisciandro GR, ed. Focused Ultrasound Techniques for the Small Animal Practitioner. John Wiley \& Sons Inc; 2014:65-79.

20. Mattoon JS, Duffy M. Spleen. In: Mattoon JS, Sellon RK, Berry CR, eds. Small Animal Diagnostic Ultrasound. 4th ed. WB Saunders; 2021:422-460

21. Lisciandro GR. Point-of-care ultrasound. In: Mattoon JS, Sellon RK, Berry CR, eds. Small Animal Diagnostic Ultrasound. 4th ed. WB Saunders; 2021:76-104.

22. Lisciandro GR. Cageside ultrasonography in the emergency room and intensive care unit. Vet Clin North Am Small Anim Pract. 2020;50(6):1445-1467.

23. Moon Larson M. Liver and spleen. In: Thrall DE, Widmer WR, eds. Textbook of Veterinary Diagnostic Radiology. 7 th ed. WB Saunders; 2018:792-822.

24. Seiler GS. Kidneys and ureters. In: Thrall DE, Widmer WR, eds. Textbook of Veterinary Diagnostic Radiology. 7th ed. WB Saunders; 2018:823-845.

25. Stieger-Vanegas SM, Frank PM. Peritoneal space. In: Thrall DE, Widmer WR, eds. Textbook of Veterinary Diagnostic Radiology. 7th ed. WB Saunders; 2018:764-791

26. Lamb CR, Hartzband LE, Tidwell AS, Pearson SH. Ultrasonographic findings in hepatic and splenic lymphosarcoma in dogs and cats. Vet Radiol. 1991;32(3):117-120.

27. Ballegeer EA, Forrest LJ, Dickinson RM, Schutten MM, Delaney FA, Young KM. Correlation of ultrasonographic appearance of lesions and cytologic and histologic diagnoses in splenic aspirates from dogs and cats: 32 cases $(2002-2005) . J$ Am Vet Med Assoc. 2007;230(5):690-696.

28. Ramirez S, Douglass JP, Robertson ID. Ultrasonographic features of canine abdominal malignant histiocytosis. Vet $R a$ diol Ultrasound. 2002;43(2):167-170.

29. Crabtree AC, Spangler E, Beard D, Smith A. Diagnostic accuracy of gray-scale ultrasonography for the detection of hepatic and splenic lymphoma in dogs. Vet Radiol Ultrasound. 2010;51(6):661-664

30. Cuccovillo A, Lamb CR. Cellular features of sonographic target lesions of the liver and spleen in 21 dogs and a cat. Vet Radiol Ultrasound. 2002;43(3):275-278.

31. Quinci M, Sabattini S, Agnoli C, Bettini G, Diana A. Ultrasonographic honeycomb pattern of the spleen in cats: correlation with pathological diagnosis in 33 cases. J Feline Med Surg. 2020;22(8):800-804.

32. Harel M, Touzet C, Barthélemy A, Ségard-Weisse EM. Prevalence and diagnostic value of the ultrasonographic honeycomb appearance of the spleen in cats. J Feline Med Surg. 2020;22(2):186-192.

33. Lux CN, Culp WTN, Mayhew PD, Tong K, Rebhun RB, Kass PH. Perioperative outcome in dogs with hemoperitoneum: 83 cases (2005-2010). J Am Vet Med Assoc. 2013;242(10):1385-1391.

34. Cudney SE, Wayne AS, Rozanski EA. Diagnostic utility of abdominal ultrasonography for evaluation of dogs with nontraumatic hemoabdomen: 94 cases (2014-2017). J Am Vet Med Assoc. 2021;258(3):290-294.

35. Wormser C, Reetz JA, Giuffrida MA. Diagnostic accuracy of ultrasound to predict the location of solitary hepatic masses in dogs. Vet Surg. 2016;45(2):208-213. 